\title{
The Presence of Mucin Increases the Anti-HIV-I Activity of the Candidate Microbicide Polyethylene Hexamethylene Biguanide (PEHMB)
}

\author{
Vanessa Pirrone ${ }^{* \neq 1}$, Shendra Miller ${ }^{1}$, Mary L Ferguson ${ }^{1}$, Lori Schlipf ${ }^{1}$, \\ Mohamed E Labib ${ }^{2}$, Robert F Rando ${ }^{2}$, Brian Wigdahl ${ }^{1}$ and Fred C Krebs ${ }^{1}$
}

\author{
Address: ${ }^{1}$ Department of Microbiology and Immunology, and Institute for Molecular Medicine and Infectious Disease, Drexel University College \\ of Medicine, Philadelphia, PA, USA and 2Novaflux Biosciences, Inc., Princeton, NJ, USA \\ Email: Vanessa Pirrone* - vanessa.pirrone@drexel.edu \\ * Corresponding author $\ddagger$ Presenting author
}

from 2005 International Meeting of The Institute of Human Virology

Baltimore, USA, 29 August - 2 September 2005

Published: 8 December 2005

Retrovirology 2005, 2(Suppl I):S95 doi:I0.I I86/1742-4690-2-SI-S95

Topical microbicides that reduce or eliminate the risk of human immunodeficiency virus type 1 (HIV-1) sexual transmission must function effectively within the cervicovaginal environment where multiple factors may impact the efficacy of the active agent. Factors relevant to potential changes in microbicide efficacy include the presence of mucins within the cervical mucus. We hypothesize that polycationic PEHMB molecules will interact with the anionic mucin molecules to form a lattice-like network that serves as a physical barrier to the movement of infectious virus and HIV-1-infected cells to the cervical and vaginal epithelia. In vitro experiments demonstrated that the anti-HIV-1 activity of PEHMB was increased almost two logs in the presence of mucin. In contrast, the activity of anionic dextran sulfate was unaffected. These results suggest that electrostatic interactions between PEHMB and mucin molecules may augment the inherent antiHIV-1 activity of PEHMB by facilitating the formation of a physical barrier between HIV-1 and susceptible cells. This property would be expected to increase the in vivo efficacy of PEHMB. 